\title{
Can physiotherapists contribute to care in the emergency department?
}

\author{
Scott F Farrell1,2
}

1. Discipline of Physiotherapy, School of Health Sciences, Faculty of Health and Medicine,

The University of Newcastle, Australia

2. Physiotherapy Department, Central Coast Local Health District, Gosford,Australia

\section{EDITORIAL}

Please cite this paper as: Farrell SF. Can physiotherapists contribute to care in the emergency department? AMJ
2014,
7,
7,
315-317.

http://doi.org/10.21767/AMJ.2014.2183

Corresponding Author:

Scott F Farrell

Discipline of Physiotherapy, School of Health Sciences, Faculty of Health and Medicine, The University of Newcastle, Callaghan 2308, NSW, Australia

Email: scott.farrell@newcastle.edu.au

\section{Introduction}

Throughout the history of healthcare practice, controversy has often surrounded instances of non-medical clinicians, such as allied health practitioners, undertaking roles traditionally performed by doctors. While this blurring of professional boundaries may occur in response to a need to enhance the efficiency of health service delivery, concerns invariably arise regarding the quality of care delivered by non-medical clinicians and the potential negative implications for patient safety. ${ }^{1}$ In recent years, physiotherapists have begun to practice in Australia's emergency departments (EDs) as autonomous, primary contact clinicians responsible for many duties traditionally solely within the domain of the medical profession. Some controversy does exist around this development, and as such, gives rise to the following questions: Do physiotherapists deliver quality care in the ED? Has the introduction of physiotherapists to ED improved service delivery as intended?

Decreasing waiting times and improving efficiency of patient management in EDs is a significant goal of modern healthcare delivery. Patients presenting with mechanical musculoskeletal pathologies can experience long waiting times in busy departments, as medical staff must prioritise higher triage category patients with more urgent medical issues. The use of suitably trained and experienced physiotherapists as primary contact clinicians has been implemented in EDs in the United Kingdom, Australia, and the United States, in an attempt to reduce waiting times for such patients, and to reduce clinical loads placed upon medical staff.

Emergency department physiotherapists (EDPs) are responsible for the management of patients with semiurgent and non-urgent (Australian triage categories 4 and 5) musculoskeletal complaints. Their role includes undertaking patient interviews and examinations, ordering investigations such as X-rays, making a diagnosis, implementing suitable interventions, referring for ongoing management, and arranging patient discharge. ${ }^{2}$ EDPs may also contribute to the care of patients with acute respiratory conditions (e.g. secretion clearance interventions) or facilitate the discharge of patients from ED into the community to reduce preventable hospital admissions.

Such clinicians practice autonomous to medical staff in the management of presentations within their scope of practice, such as ankle or knee sprains and mechanical back pain, in a similar capacity as a physiotherapist working in a private clinic. EDPs may also practice in collaboration with medical staff in the management of simple limb fractures, or as a secondary practitioner providing physiotherapy input to respiratory conditions or an opinion regarding musculoskeletal injuries. In Australia, as this role is outside the traditional duties of physiotherapists, this is termed "advanced practice". ${ }^{3}$

\section{History and emergence of EDPs}

Prior to the 1970s, physiotherapists practiced under the direct supervision of their medical colleagues, requiring a doctor's referral to treat a patient. However, in 1976, the profession in Australia began to practice as primary contact healthcare providers, ${ }^{4}$ autonomously assessing and treating appropriate musculoskeletal conditions, such as joint sprains or mechanical spinal pain, while still working in close consultation with medical practitioners. EDPs first emerged in the United Kingdom ${ }^{5,6}$ with the role subsequently spreading to Australia, where it continues to 
evolve today as a strategy to reduce patient waiting times, in line with the National Emergency Access Target (NEAT). ${ }^{\text {a }}$ However, after physiotherapy's formative years entering this new clinical environment, a number of questions may be considered: First, as EDPs are undertaking some clinical duties traditionally performed by medical doctors, is there evidence that these practitioners are delivering quality patient care? Second, does the implementation of EDPs successfully reduce waiting times or contribute positively to ED operation?

\section{EDPs and patient outcomes}

EDPs bring their skills and knowledge of musculoskeletal clinical assessment and diagnosis, as well as various techniques specific to the discipline such as manual therapy, exercise prescription, strapping and taping, and prescription of mobility aids. Healthcare outcomes for patients managed by these clinicians in ED have only been investigated by a limited number of studies, ${ }^{7}$ but research findings have generally been encouraging. Management of peripheral soft tissue injuries by EDPs is of appropriate quality, as evidenced by comparable clinical outcomes (such as pain, function, and representation rate) with that of patients managed in the traditional ED model. ${ }^{8,9}$ Jibuike et al. ${ }^{10}$ commented on the high diagnostic accuracy of an EDP working in a specialised role managing acute knee injuries in collaboration with a knee surgeon, and Lau et al. ${ }^{11}$ found patients suffering low back pain managed in ED by a physiotherapist reported significantly lower levels of pain on discharge than those without EDP input.

Patient satisfaction with their management by EDPs has been reported as higher than those managed in the traditional model for musculoskeletal injuries ${ }^{8,12}$ and for mechanical lower back pain. ${ }^{11}$ Interestingly, however, one study noted a significantly greater time period for return to usual activity for patients with soft tissue injuries managed by EDPs, ${ }^{12}$ so it can be seen that the clinical effectiveness of physiotherapy in ED requires attention and deliberation in some contexts.

\section{EDPs and healthcare service outcomes}

Much of the rationale for the introduction and development of the EDP role has been related to reducing ED waiting times and improving service efficiency. It is, therefore, surprising that very few studies have actually examined the effectiveness of EDP practice from a service delivery perspective. There are reports from some studies that the addition of an EDP to an ED service can lead to decreased waiting times and length of stay, ${ }^{9}$ reduced time demands on medical staff, ${ }^{10}$ and more efficient referral and access to outpatient physiotherapy treatment when indicated. ${ }^{6}$ A large, randomised single centre trial by Richardson et al. ${ }^{12}$ found management of musculoskeletal pathologies by EDPs to be equivalent in cost to the management of such patients by ED medical staff.

Reduction of preventable hospital admissions, by facilitating the discharge of patients with mobility deficits, is another theorised potential benefit of EDP input to patient management in ED. The effectiveness of EDPs at achieving this aim has only been examined in one study, ${ }^{13}$ which found that physiotherapist intervention to improve mobility in patients admitted to an ED extended care unit ${ }^{b}$ did not reduce admission rates for the unit.

\section{Challenges to consider}

Comprehensive assessment of the strengths and weaknesses of current EDP practice, as well as whether the current ED physiotherapy model is achieving the desired goal of enhancing ED operation, is significantly limited by a lack of suitably designed research. It is important to note as well that EDP roles are intended to be undertaken by experienced musculoskeletal physiotherapists that have undertaken further training in basic orthopaedics and radiology as applicable to the ED, to ensure that a high standard of clinical care and patient safety are maintained. This means that not every physiotherapist is suitable for the role, and as such for the role to continue to develop and improve, appropriate training opportunities and quality assurance measures must be in place in health services implementing ED physiotherapy.

It should be reiterated that physiotherapists in Australia are licensed to practice as autonomous primary healthcare practitioners in the management of musculoskeletal pathology that falls within the scope of the profession, such as joint sprains and mechanical spinal pain. This primary contact status, which has existed since 1976, forms the model of practice by which private community physiotherapy clinics operate, and it is the transferral of this model of practice to the emergency context, with appropriate training and quality assurance measures, that is the basis of the EDP clinical role.

\footnotetext{
${ }^{b}$ ED extended care units, also known as short stay units, provide an extended duration of care for patients requiring a longer period of observation or intervention than that usually offered in ED, but not requiring hospital admission. ${ }^{8}$
}

${ }^{\mathrm{a}} \mathrm{NEAT}$ is an Australian federal initiative that seeks to reduce ED waiting times, such that by 2015,90 per cent of patients will be admitted, referred, or discharged from ED within four hours of presentation. 
Ensuring that patients managed by physiotherapists fall within the scope of practice of the profession is a significant responsibility for physiotherapists working in a primary contact capacity, in both the context of emergency or in a private community clinic. Awareness of which cases can be safely managed by a physiotherapist is important for EDPs, and to ensure patient safety from a service delivery perspective, clear guidelines should exist for appropriate selection of patients by EDPs, as well as the transferral of care to an ED medical officer should a patient's requirements extend beyond the scope of the physiotherapy profession.

\section{Conclusion}

The inclusion of physiotherapists in ED as primary contact treating practitioners could be seen as a positive acquisition for the ED clinical team; EDPs have a unique set of skills to apply to patient management that appear to benefit patients accessing the health service, and may improve the overall service delivery in this department. While EDPs are undertaking some clinical duties traditionally performed by medical staff, their inclusion as members of the team in ED should not be seen as contrary to the medical profession's leadership in this domain, but rather as an adjunct to the current clinical services offered in ED. As the medical and physiotherapy professions progress in response to modern Australia's ever-changing healthcare needs, blurring of professional boundaries will likely continue to occur. As such, the impact of these developments upon patient outcomes and health service delivery must be explored in order to ensure continued improvement of healthcare delivery.

\section{References}

1. Jiwa M. When only a doctor will do. Australas Med J. 2012;5(7):414-7. doi:10.4066/AMJ.2012.1446.

2. Anaf S, Sheppard L. Physiotherapy as a clinical service in emergency departments: a narrative review. Physiother. 2007;93(4):243-52.

3. Anaf S. Physiotherapy's role in emergency department settings: a qualitative investigation of emergency stakeholders' perceptions. Townsville: James Cook University; 2008.

4. Kruger J. Patient referral and the physiotherapist: three decades later. J Physiother. 2010;56:217-8.

5. Kempson S. Physiotherapy in an accident and emergency department. Accid Emerg Nurs. 1996;4(4):198-202.

6. Morris $C$, Hawes $S$. The value of accident and emergency based physiotherapy services. J Accid Emerg Med. 1996 Mar;13(2):111-3.

7. Kilner E. What evidence is there that a physiotherapy service in the emergency department improves health outcomes? A systematic review. J Health Serv Res Policy. 2011;16(1):51-8.
8. McClellan C, Greenwood R, Benger J. Effect of an extended scope physiotherapy service on patient satisfaction and the outcome of soft tissue injuries in an adult emergency department. Emerg Med J. 2006;23(5):384-7.

9. Taylor N, Norman E, Roddy L, Tang C, Pagram A, Hearn K. Primary contact physiotherapy in emergency departments can reduce length of stay for patients with peripheral musculoskeletal injuries compared with secondary contact physiotherapy: a prospective non-randomised controlled trial. Physiother. 2011;97:107-14.

10. Jibuike O, Paul-Taylor G, Maulvi S, Richmond P, Fairclough J. Management of soft tissue knee injuries in an accident and emergency department: the effect of the introduction of a physiotherapy practitioner. Emerg Med J. 2003;20(1):37-9.

11. Lau PM-Y, Chow DH-K, Pope M. Early physiotherapy intervention in an Accident and Emergency Department reduces pain and improves satisfaction for patients with acute low back pain: a randomised trial. Aust J Physiother. 2008;54:243-9.

12. Richardson B, Shepstone L, Poland F, Mugford M, Finlayson B, Clemence N. Randomised controlled trial and cost consequences study comparing initial physiotherapy assessment and management with routine practice for selected patients in an accident and emergency department of an acute hospital. Emerg Med J. 2005;22(2):87-92.

13. Jesudason C, Stiller K, McInnes M, Sullivan T. A physiotherapy service to an emergency extended care unit does not decrease admission rates to hospital: a randomised trial. Emerg Med J. 2012;29(8):664-9.

\section{PEER REVIEW}

Peer reviewed.

\section{CONFLICTS OF INTEREST}

The author declares no conflict of interest. 